\title{
Rotational Diffusion of Macromolecules and Nanoparticles Modeled as Non-Overlapping Bead Arrays in an Effective Medium
}

\author{
Hengfu Wu, Umar Twahir, Alishia Davis, Ebenezer Duodo, Bahareh Kashani, Young Lee, \\ Cindy Pena, Noni Whitley and Stuart A. Allison * \\ Department of Chemistry, Georgia State University, P.O. Box 4098, Atlanta, GA 30302, USA; \\ E-Mails: hwu5@student.gsu.edu (H.W.); utwahir1@student.gsu.edu (U.T.); \\ adavis103@student.gsu.edu (A.D.); eduodu1@ student.gsu.edu (E.D.); \\ bkashani1@student.gsu.edu (B.K.); ylee3@ @student.gsu.edu (Y.L.); \\ cpena2@student.gsu.edu (C.P.); nwhitley2@student.gsu.edu (N.W.) \\ * Author to whom correspondence should be addressed; E-Mail: sallison@ gsu.edu; \\ Tel.: +1-404-413-5519.
}

Received: 21 March 2011; in revised form: 26 April 2011 / Accepted: 11 May 2011 / Published: 13 May 2011

\begin{abstract}
In this work, the retarding influence of a gel on the rotational motion of a macromolecule is investigated within the framework of the Effective Medium (EM) model. This is an extension of an earlier study that considered the effect of a gel on the translational motion of a macromolecule [Allison, S. et al. J. Phys. Chem. B 2008, 112, 5858-5866]. The macromolecule is modeled as an array of non-overlapping spherical beads with no restriction placed on their size or configuration. Specific applications include the rotational motion of right circular cylinders and wormlike chains modeled as strings of identical touching beads. The procedure is then used to examine the electric birefringence decay of a 622 base pair DNA fragment in an agarose gel. At low gel concentration $(M \leq 0.010 \mathrm{gm} / \mathrm{mL}$ ), good agreement between theory and experiment is achieved if the persistence length of DNA is taken to be $65 \mathrm{~nm}$ and the gel fiber radius of agarose is taken to be $2.5 \mathrm{~nm}$. At higher gel concentrations, the EM model substantially underestimates the rotational relaxation time of DNA and this can be attributed to the onset of direct interactions that become significant when the effective particle size becomes comparable to the mean gel fiber spacing.
\end{abstract}


Keywords: rotational diffusion; gel diffusion; electric birefringence; DNA dynamics

\section{Introduction}

The subject of biomolecular transport in congested media is of vital interest in such diverse subjects as drug delivery across membranes and the sieving action of a gel in electrophoresis. In particular, diffusion (translational and rotational) has been studied in a wide range of environments including the cytoplasm of cells [1], concentrated suspensions [2], gels or hydrogels [3-13], and mucus [14]. The diffusion of a host particle through a rigid gel matrix is reduced, relative to diffusion in "free solution", by long range hydrodynamic interaction and short range steric effects. For translational diffusion, these two effects can be considered separately [3,15-17]. A simple way of dealing with the contribution of long range hydrodynamic interaction makes use of the Effective Medium (EM) model originally developed by Brinkman [18], and Debye and Bueche [19].

In the EM model, the "fluid" surrounding the particle is treated as a hydrodynamic continuum, and includes both solvent and the "gel" support medium. A special screening term is added to the external force/volume on the fluid in the low Reynolds number Navier-Stokes equation that accounts for the presence of a gel. The resulting equation is what we call the Brinkman equation. Starting from a microscopic model, Felderhof and Deutch were able to derive the Brinkman equation as a mean field approximation [20]. The EM model has been applied to translational diffusion [3,4,16,21], rotational diffusion [20], electrophoresis [15,22], and the electrophoretic stretch of duplex DNA in gels [23,24].

The principle objective of the present work is to extend our earlier study of the translational diffusion of a macromolecule modeled as an array of non overlapping beads in an EM to the case of rotational diffusion. In Section 2.1, the Brinkman equation is introduced and the rotation of a single sphere in an EM is discussed. This is then extended to the more complex problem of an array of non-overlapping beads. In Section 2.2, we focus first on the parameterization of a linear string of touching beads and later extend that to a wormlike chain model. The wormlike chain model is relevant to modeling the electric birefringence or dichroism decay of duplex DNA [12] which is also discussed in Section 2 and again in Section 4. In Section 3.1, the rotational diffusion of some simple bead arrays in the presence and absence of a gel to illustrate the accuracy of the methodology employed as well the influence long range hydrodynamic interaction has rotational diffusion. In Section 3.2, this is applied to the rotational diffusion of a $622 \mathrm{bp}$ DNA fragment in a gel [12]. Here, we are able to compare EM modeling with rotational diffusion in agarose gel measured by electric birefringence. For dilute gels ( $\leq 0.01 \mathrm{gm}$ dry gel $/ \mathrm{mL}$ solvent), we obtain good agreement between modeling and experiment for reasonable values of model parameters. For more concentrated gels, model rotational relaxation times substantially underestimate experimental relaxation times. This, however, is believed to be due to the neglect of steric interactions in modeling that become important at high gel concentration. In Section 4, the principle conclusions of the present work are summarized. 


\section{Results and Discussion}

\subsection{Transport Theory of Bead Arrays in an Effective Medium}

In the Effective Medium, EM, model, the fluid is assumed to obey the Brinkman [18] and solvent incompressibility equations defined by

$$
\begin{gathered}
\eta \nabla^{2} \underline{v}(\underline{r})-\underline{\nabla} p(\underline{r})=\eta \lambda^{2} \underline{v}(\underline{r}) \\
\underline{\nabla} \cdot \underline{v}(\underline{r})=0
\end{gathered}
$$

Where $\eta$ is the solvent viscosity, $\underline{v}(\underline{r})$ is the local fluid velocity at point $\underline{r}, p$ is the local pressure, and $\lambda$ (units of $1 /$ length) is the gel screening parameter. This parameter can be related to the gel concentration, $M$, (in gm dry gel material per gm of solvent) and gel fiber radius, $r_{f}$, by the relation $[16,25]$

$$
\frac{1}{\lambda^{2}}=-\frac{3 \rho_{g} \omega_{s} r_{f}^{2}}{20 M}\left[\ln \left(\frac{M}{\rho_{g} \omega_{s}}\right)+0.931\right]
$$

In Equation (3), $\rho_{g}$ denotes the mass density of dry gel (which equals $1.64 \mathrm{gm} / \mathrm{mL}$ for agarose [26]), and $\omega_{s}$ denotes the ratio of dry gel volume to hydrated gel volume (which equals 0.625 for agarose [27]). The term on the right hand side of Equation (1) represents an external force/unit volume due to the viscous drag on the fluid produced by the presence of the gel. In general, other external forces on the fluid may be present as well. This is particularly true in modeling the transport of macroions in external electric fields (electrophoresis) [28]. However, the cases of interest in the present work involve rotational relaxation in the absence of an external electric field. Under these conditions, the additional external force terms can be ignored for a good approximation.

For later reference, it will be useful to consider the local fluid velocity and pressure of a spherical particle of radius " $a$ " rotating about its center with angular velocity $\underline{\omega}$ in an EM that is at rest far from the particle. In this case it is straightforward to solve Equations (1) and (2) and obtain $p(\underline{r})=$ constant and

$$
\underline{v}(\underline{r})=\left(\frac{a}{r}\right)^{3}\left(\frac{1+\lambda r}{1+\lambda a}\right) e^{-\lambda(r-a)}(\underline{\omega} x \underline{r})
$$

In Equation (4), " $x$ " denotes the vector cross product. Note that the fluid velocity falls off rapidly moving away from the rotating sphere. The local stress tensor, $\underline{\underline{\sigma}}(\underline{r})$, is related to the velocity and pressure by $[29,30]$

$$
\underline{\underline{\sigma}}(\underline{r})=-p(\underline{r}) \underline{\underline{I}}+\eta\left(\underline{\underline{\nabla v}}+\underline{\underline{\nabla v^{T}}}\right)
$$

Where $\underline{\underline{I}}$ is the 3 by 3 identity tensor $\left((\underline{\underline{I}})_{\mathrm{jk}}=\delta_{j k}\left(\delta_{j k}\right.\right.$ is the Kronecker delta)), $(\underline{\underline{\nabla v}})_{\mathrm{jk}}=\nabla_{j} v_{k}(\underline{r})$, and $\left(\underline{\underline{\nabla v^{T}}}\right)_{j k}=\nabla_{k} v_{j}(\underline{r})$. For a point $\underline{r}=a \underline{n}(\underline{r})$ on the surface of the sphere $(\underline{n}(\underline{r})$ is a local outward (into the fluid) normal to the sphere), the local force/area exerted by the sphere of the fluid, $\underline{f}(\underline{r})$, is related to the local stress by

$$
\underline{f}(\underline{r})=-\underline{\sigma}(\underline{r}) \cdot \underline{n}(\underline{r})=\eta \gamma(\lambda a)(\underline{\omega} \times \underline{n}(\underline{r}))
$$




$$
\gamma(\lambda a)=\frac{\left(3+3 \lambda a+\lambda^{2} a^{2}\right)}{(1+\lambda a)}
$$

If the sphere were rotating as a rigid body about some point different from its center, then $\underline{f}(\underline{r})$ would be different. Let $r^{*}$ denote the position of the center of the sphere relative to the center of rotation. For a good approximation, we can write

$$
\underline{f}(\underline{r}) \cong \frac{F}{S}+\eta \gamma(\lambda a)(\underline{\omega} \times \underline{n}(\underline{r}))
$$

In Equation (8), $\underline{F}$ denotes the net instantaneous force exerted by the bead on the fluid and $S=4 \pi a^{2}$ is the surface area of the bead. The net force, in turn, equals $6 \pi \eta a\left(\underline{\omega} x \underline{r^{*}}\right)$.

In a recent analysis employing the Boundary Element method, a general expression was derived for the local fluid velocity of an array of non overlapping beads translating with uniform velocity through an Effective Medium that obeys Equations (1,2) [16]. It is straightforward to extend that analysis to the rotation of a bead array. As illustrated in Figure 1, the macromolecule is modeled as an array of $N$ non overlapping beads in which their radii, $\left\{a_{j}\right\}$, and relative positions are arbitrary. Let $\underline{r}$ denote the center of rotation of the rigid body bead array, $\underline{\omega}$ the angular velocity of the bead array, and $\underline{x}_{J}$ and $a_{\mathrm{J}}$ the centroid position vector and radius of bead $J$. The fluid is assumed to be at rest far from the array. It shall also be assumed that for a point, $\underline{r}$, on the surface of bead $J$, we can write

$$
\underline{f}(\underline{r}) \cong \frac{\underline{F}_{J}}{S_{J}}+\eta \gamma\left(\lambda a_{J}\right)(\underline{\omega} \times \underline{n}(\underline{r}))
$$

Where $\underline{F}_{J}$ is the net force exerted by bead $\mathrm{J}$ on the fluid and $S_{J}$ is the corresponding surface area. The second term on the right hand side of Equation (9) is similar to the "Volume Correction" approach of Garcia de la Torre and Rodes [31].

Figure 1. Array of $N$ Non Overlapping Beads. The bead radii, $\left\{a_{j}\right\}$, and the position of the bead centers is arbitrary.

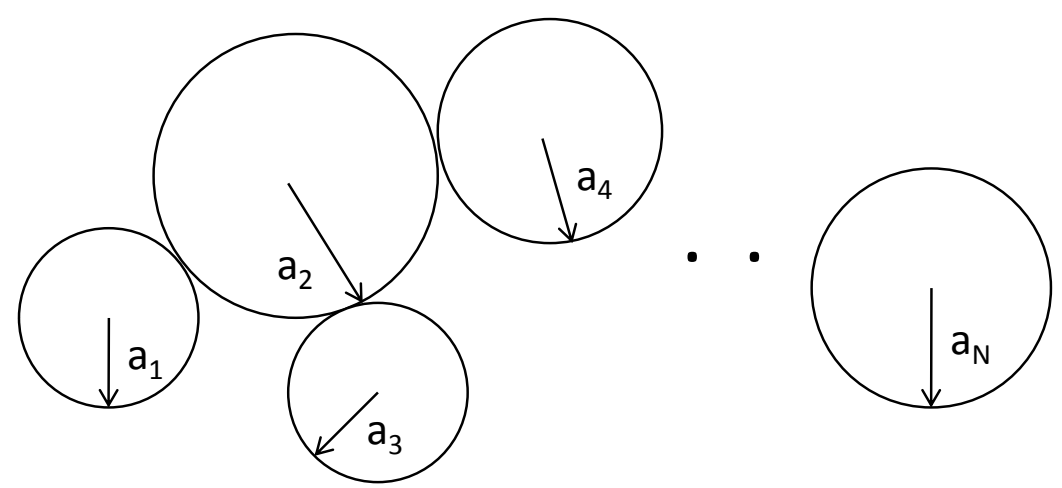

It is worthwhile to briefly discuss the physical basis of Equation (9). Hydrodynamic interaction, HI, between two beads arises as a consequence of their relative motion through a viscous fluid. In the absence of a gel, the disturbance of the fluid velocity produced by one bead, $J$, centered at $\underline{r}_{J}$, at the site of a second bead, $K$, centered at $\underline{r}_{K}$ separated by distance $r_{J K}=\left|\underline{r}_{J K}\right|=\left|\underline{r}_{J}-\underline{r}_{K}\right|$, falls off as $1 / r_{J K}$ if their centers are in relative motion $[29,30]$. On the other hand, if their centers are not in relative 
motion, but if one or both beads are rotating about their centers, then the disturbance falls off as $1 / r_{J K}{ }^{3}$, which is clear from Equation (4). The presence of a gel modifies these distance dependent interactions, but the important point is that long range $\mathrm{HI}$ is determined primarily by the relative translational motion of the bead centers. The dominant long range HI between the beads is contained implicitly in the first term on the right hand side of Equation (9). The second term reflects the fluid stress arising from the rotation of bead $J$ itself relative to the fluid. It should be emphasized that Equation (9) is approximate and ignores shorter range $\mathrm{HI}$ interactions.

Following our earlier analysis [16] of a translating bead array, the corresponding results for a rotating bead array can be written

$$
\underline{\omega} x\left(\underline{r}_{K}-\underline{r}^{*}\right)=\sum_{J=1}^{N}\left[\underline{\underline{C}}_{K J} \cdot\left(\underline{r}_{J}-\underline{r}^{*}\right)+\frac{1}{\zeta_{J}} \underline{\underline{H}}_{K J} \cdot \underline{F}_{J}\right]+\sum_{J=1}^{N} \underline{\omega} x \underline{p}_{K J}
$$

Where $\zeta_{J}=6 \pi \eta a_{J}$

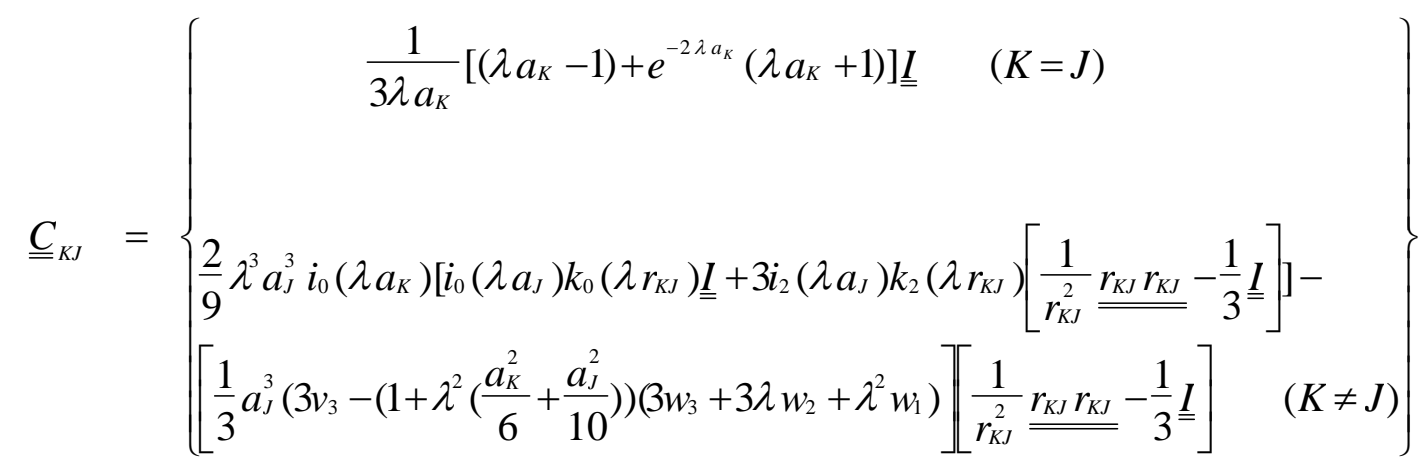

$$
\begin{aligned}
& \underline{\underline{H}}_{K J}=\left\{\begin{array}{cc}
i_{0}\left(\lambda a_{K}\right) e^{-\lambda a_{K}} \underline{\underline{I}} \quad(K=J) \\
\lambda a_{J} i_{0}\left(\lambda a_{K}\right) i_{0}\left(\lambda a_{J}\right) k_{0}\left(\lambda r_{K J}\right) \underline{\underline{I}}+ \\
\frac{3 a_{J}}{2 \lambda^{2}}\left[3 v_{3}-\left(1+\frac{\lambda^{2}\left(a_{J}^{2}+a_{K}^{2}\right)}{6}\right)\left(3 w_{3}+3 \lambda w_{2}+\lambda^{2} w_{1}\right)\right]\left[\frac{1}{r_{K J}^{2}} \underline{\underline{r_{K J}}} r_{K J}-\frac{1}{3} \underline{\underline{I}}\right] & (K \neq J)
\end{array}\right\} \\
& w_{n}=\frac{e^{-\lambda r_{K J}}}{r_{K J}^{n}} \\
& v_{n}=\frac{1}{r_{K J}^{n}} \\
& \underline{p}_{K J}=i_{0}\left(\lambda a_{K}\right)\left(\frac{a_{J}}{r_{K J}}\right)^{3} e^{-\lambda\left(r_{K J}-a_{J}\right)}\left(\frac{1+\lambda r_{K J}}{1+\lambda a_{J}}\right)\left(\underline{r}_{K}-\underline{r}_{J}\right)
\end{aligned}
$$

In Equations (11,12), $i_{n}$ and $k_{n}$ are modified spherical Bessell functions. Specifically, $i_{0}(z)=\sinh (z) / z, i_{2}(z)=\sinh (z) / z-3 \cosh (z) / z^{2}+3 \sinh (z) / z^{3}, k_{0}(z)=e^{-z} / z, k_{2}(z)=e^{-z}\left(1 / z+3 / z^{2}+3 / z^{3}\right)$. Also, $\left(\underline{\underline{r_{K J} r_{K J}}}\right)_{j k}=\left(\underline{r}_{K}-\underline{r}_{J}\right)_{j}\left(\underline{r}_{K}-\underline{r}_{J}\right)_{k}$.

The typical procedure followed in a resistance problem [29,30,32-34], as applied to the more general problem in an EM, is to compute the elements of $\underline{\underline{C}}_{K J}$ and $\underline{\underline{H}}_{K J}$ once the geometry of a bead 
array and conditions of the EM are defined. The bead array is then rotated about three orthogonal axes with unit angular velocity,

$$
\underline{\omega}^{(p)}=\underline{e}_{p}
$$

Where $\underline{e}_{p}$ is a unit vector along axis $p(p=1,2$, or 3$)$ in some convenient frame of reference. Let $\underline{F}_{J}^{(p)}$ denote the net force exerted by bead $J$ on the fluid when the array is rotated about axis $p$. Then Equation (10) can be written

$$
\begin{gathered}
\sum_{J=1}^{N} \frac{1}{\zeta_{J}} \underline{\underline{H}}_{K J} \cdot \underline{F}_{J}^{(p)}=\underline{A}_{K}^{(p)} \\
\underline{A}_{K}^{(p)}=\underline{e}_{p} x\left(\underline{r}_{K}-\underline{r}^{*}\right)-\sum_{J=1}^{N}\left[\underline{C}_{K J} \cdot\left(\underline{e}_{p} x\left(\underline{r}_{J}-\underline{r}^{*}\right)\right)+\underline{e}_{p} x \underline{p}_{K J}\right]
\end{gathered}
$$

It is helpful to view $\underline{F}_{J}^{(p)}$ and $\underline{A}_{K}^{(p)}$ as 3 by 1 column vectors. From these, we can define $3 \mathrm{~N}$ by 3 super matrices, $\underline{\underline{F}}$ and $\underline{\underline{A}}$, by

$$
\underline{F}=\left(\begin{array}{ccc}
\underline{F}_{1}^{(1)} & \underline{F}_{1}^{(2)} & \underline{F}_{1}^{(3)} \\
\underline{F}_{2}^{(1)} & \underline{F}_{2}^{(2)} & \underline{F}_{2}^{(3)} \\
\vdots & \vdots & \vdots \\
\underline{F}_{N}^{(1)} & \underline{F}_{N}^{(2)} & \underline{F}_{N}^{(3)}
\end{array}\right)
$$

The super matrix, $\underline{\underline{A}}$, has a very similar form to Equation (19) above. Also, the $\underline{\underline{H}}_{K J}$ terms defined by

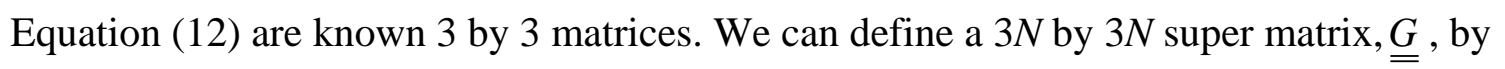

$$
\underline{\underline{G}}=\left(\begin{array}{cccc}
\underline{\underline{H}}_{11} / \zeta_{1} & \underline{\underline{H}}_{12} / \zeta_{2} & \cdots & \underline{\underline{H}}_{1 N} / \zeta_{N} \\
\underline{\underline{H}}_{21} / \zeta_{1} & \underline{\underline{H}}_{21} / \zeta_{2} & \cdots & \underline{\underline{H}}_{2 N} / \zeta_{N} \\
\vdots & \vdots & \ddots & \vdots \\
\underline{\underline{H}}_{N 1} / \zeta_{1} & \underline{\underline{H}}_{N 2} / \zeta_{2} & \cdots & \underline{\underline{H}}_{N N} / \zeta_{N}
\end{array}\right)
$$

In compact notation, Equation (16) can be written

$$
\underline{\underline{G}} \cdot \underline{\underline{F}}=\underline{\underline{A}}
$$

The matrix, $\underline{\underline{G}}$, is invertible and let $\underline{\underline{G}}^{-1}$ denote the inverse. Then

$$
\underline{\underline{F}}=\underline{\underline{G}}^{-1} \cdot \underline{\underline{A}}
$$

A very similar relation was derived previously for a bead array translating with uniform velocity, $\underline{u}$.

Before it is possible to compute various resistance tensors from modeling, it is necessary to compute the total force, $\underline{F}_{t o t}$, and total torque, $\underline{T}_{t o t}\left(\underline{r}^{*}\right)$, exerted by the bead array on the fluid if it is translated with velocity,$\underline{u}$, and rotated with angular velocity, $\underline{\omega}$, about some point, $\underline{r}^{*}$, in the fluid which is at rest far from the array. In the present work, $\underline{r}^{*}$ is chosen as the center of mass of the bead array. The total force and torque can be written [35]

$$
\begin{gathered}
\underline{F}_{t o t}=\underline{\Xi}_{T} \cdot \underline{u}+\underline{\Xi}_{C}^{T}\left(\underline{r}^{*}\right) \cdot \underline{\omega} \\
\underline{T}_{t o t}\left(\underline{r}^{*}\right)=\underline{\Xi}_{C}\left(\underline{r}^{*}\right) \cdot \underline{u}+\underline{\Xi}_{R}\left(\underline{r}^{*}\right) \cdot \underline{\omega}
\end{gathered}
$$


Where $\underline{\Xi}_{T}, \underline{\Xi}_{R}\left(\underline{r}^{*}\right)$, and $\underline{\Xi}_{C}\left(\underline{r}^{*}\right)$ denote translation, rotation, and coupling resistance tensors, respectively. Quantities with argument " $\underline{r} *$ " depend on the choice of $\underline{r}^{*}$. Also, the " $T$ " superscript on the coupling tensor in Equation (23) denotes transpose. $\underline{F}_{t o t}$ and $\underline{T}_{t o t}\left(\underline{r}^{*}\right)$ can be obtained from modeling by the relations

$$
\begin{aligned}
& \underline{F}_{t o t}=\sum_{J=1}^{N} \int_{S_{J}} d S_{r} \underline{f}(\underline{r})=\sum_{J=1}^{N} \underline{F}_{J} \\
& \underline{T}_{t o t}\left(\underline{r}^{*}\right)=\sum_{J=1}^{N} \int_{S_{J}} d S_{r}\left(\underline{r}-\underline{r}^{*}\right) x \underline{f}(\underline{r})
\end{aligned}
$$

For a bead array that is not translating $(\underline{u}=\underline{0})$, but is rotating with angular velocity $\underline{e}_{p}$ about $\underline{r}^{*}$,

$$
\underline{T}^{(p)}\left(\underline{r}^{*}\right)=\sum_{J=1}^{N}\left[\left(\underline{r}_{J}-\underline{r}^{*}\right) x \underline{F}_{J}^{(p)}+\frac{8 \pi a_{j}^{3}}{3} \eta \gamma\left(\lambda a_{J}\right) \underline{e}_{p}\right]
$$

From Equations $(23,24)$, the net force and net torque give the $\mathrm{p}$-th row of $\underline{\underline{\Xi}}_{C}\left(\underline{r}^{*}\right)$, and $p$-th column of $\underline{\Xi}_{R}\left(\underline{r}^{*}\right)$, respectively. The components of $\Xi_{T}$ are obtained by translating the array along three orthogonal axes, computing the net forces, and then using Equation (23).

The connection between the resistance tensors and corresponding mobility or diffusion tensors is well known [29,30,35,36]. The origin dependent translational diffusion tensor, $\underline{\underline{D}}_{T}\left(\underline{r}^{*}\right)$, and origin independent rotational diffusion tensor, $\underline{\underline{D}}_{R}$, are given by

$$
\begin{gathered}
\underline{\underline{D}}_{T}\left(\underline{r}^{*}\right)=k_{B} T\left[\underline{\underline{\Xi}}_{T}-\underline{\underline{\Xi}}_{C}^{T}\left(\underline{r}^{*}\right) \cdot \underline{\underline{\Xi}}_{R}^{-1}\left(\underline{r}^{*}\right) \cdot \underline{\underline{\Xi}}_{C}\left(\underline{r}^{*}\right)\right]^{-1} \\
\underline{\underline{D}}_{R}=k_{B} T \underline{\Xi}_{R}^{-1} \\
\underline{\Xi}_{R}=\left[\underline{\Xi}_{R}\left(\underline{r}^{*}\right)-\underline{\Xi_{C}}\left(\underline{r}^{*}\right) \cdot \underline{\Xi}_{T}^{-1} \cdot \underline{\Xi}_{C}^{T}\left(\underline{r}^{*}\right)\right]
\end{gathered}
$$

In modeling, the origin independent rotational friction tensor, $\Xi_{R}$, can be diagonalized and let $\Lambda_{n}{ }^{\prime}$ ( $n=1,2$, or 3 ) denote the $n$-th eigenvalue. We shall define the eigenvalues such that $\Lambda_{1}{ }^{\prime} \geq \Lambda_{2}{ }^{\prime} \geq \Lambda_{3}$. Also define the reduced dimensionless eigenvalue for an array of $N$ identical beads of radius $a$,

$$
\Lambda_{n}=\frac{\Lambda_{n}^{\prime}}{8 \pi \eta a^{3} N}
$$

The denominator in Equation (31) represents the rotational friction factor of a sphere of volume equal to that of our bead array.

The modeling results of the present work shall be given in terms of these dimensionless eigenvalues. These, in turn, can be related to the eigenvalues of $\underline{\underline{\Xi}}_{R}$ and $\underline{\underline{D}}_{R}$ through Equations $(29,31)$. We also want to relate these to the longest lifetime, $\tau_{l}$, of the "off-field" electric birefringence decay of a dilute solution of macromolecules. We can write [12,37]

$$
\tau_{l}=\frac{1}{6 D_{R 1}}=\frac{4 \pi \eta a^{3} N}{3 k_{B} T} \Lambda_{1}
$$




\subsection{Modeling the Rotation of Linear Macromolecules as Strings of Identical Touching, but} Non-Overlapping Beads

A key element in the parameterization of the bead radii of our "coarse grained" models is to find arrays of identical beads that are able to reproduce the rotational friction, $\Xi_{R}$, or diffusion, $\underline{\underline{D}}_{R}$, tensor of the actual structure to considerable accuracy. From Section 2.1, this is equivalent to matching the reduced eigenvalues, $\left\{\Lambda_{n}\right\}$. Furthermore, since experiments such as electric birefringence [12,37] or dichroism $[38,39]$ are sensitive to particular eigenvalues $\left(\Lambda_{l}\right.$ for example), then we can focus on matching that particular eigenvalue.

This shall be illustrated for the special case of a right circular cylinder of length $L$ and axial radius, $R$. For the right circular cylinder in an EM with $\lambda=0$ [40]

$$
\begin{gathered}
\Lambda_{1}^{r o d}(L, R)=\frac{L^{2}}{18 R^{2}\left[\ln (p)+\delta_{\perp}(p)\right]} \\
p=\frac{L}{2 R} \\
\delta_{\perp}(p)=-0.662+0.917 / p-0.050 / p^{2}
\end{gathered}
$$

For the corresponding array made up of a linear string of $N$ touching beads of radius $a$, we shall set

$$
L=2 a(N-c)
$$

In Equation (36), $a$ and $c$ are left as adjustable parameters but it is assumed that $a$ is proportional to $R$. For an array made up of $N$ beads, $\Lambda_{l}$ from Equation (31) is independent of $\eta$ and $a$, and is computed by the procedure described in Section 2.1. The quantity $\Lambda_{l}{ }^{\text {rod }}$ from Equation (33) is independent of $\eta$ and only depends on the ratio, $L / R$, or equivalently $L / a$. For assumed values of $a$ and $c$, Equation (36) then gives us a direct correspondence between $L, N, a$, and $c$. It is straightforward to construct an Excel spreadsheet in which $a$ and $c$ are defined as input parameters. $\Lambda_{l}^{\text {rod }}$ and $\Lambda_{l}$ are compared for a range of $N$ values. (In the present work, we are interested in long rods and $N$ is varied from 20 to 100 ). In the fitting procedure, we start by setting $c$ equal to 0 and then vary $a$ until the sum of the square of the differences, SR, between $\Lambda_{l}^{\text {rod }}$ and $\Lambda_{l}$ is minimized. Then, $c$ is incremented by a small amount and the procedure is repeated. This process is continued until that combination of $a$ and $c$ is found that minimizes SR overall. For a good approximation, this is given by $c=0.20$ and $a / R=1.20$.

We can apply this duplex DNA made up of $n_{b p}$ base pairs and $L$ (in nm) $=0.34 n_{b p}$. Also, $R$ is $1.0 \pm 0.1$ [41] and this parameter shall be set equal to $1.0 \mathrm{~nm}$ in the present work. This model is very similar to that of Hagerman and Zimm [42] and involves minor corrections in the choice of the $a$ and $c$ parameters. Unless the duplex DNA is very short, however, it is better to model the DNA as a wormlike chain of persistence length $P$ (contour length equal to $L$ and axial radius equal to $R$ ) rather than a right circular cylinder. For DNA, $P$ is typically in the $50 \mathrm{~nm}$ size range, but this varies with ionic strength $[43,44]$. As an illustrative example, $L=211.5 \mathrm{~nm}$ for $622 \mathrm{bp}$ DNA which is more than 4 persistence lengths long. Also, for $a=1.206$ and $c=0.20$, Equation (36) requires $N$ be set to 88 . For DNA fragments of this size, a rigid rod model is inadequate. Fortunately, it is straightforward to generalize the "linear string" model of the previous paragraph to a "discrete wormlike chain" model that has been widely used in the past $[42,45]$. Let $\langle\cos \theta\rangle=\left\langle\underline{e}_{J} \cdot \underline{e}_{J+1}\right\rangle$ where $\underline{e}_{J}$ denotes the unit 
vector along the $J$-th virtual bond of our discrete wormlike chain model and brackets denote an ensemble or long time average. If the distance between adjacent beads is $2 a$, then $[42,43,45]$

$$
P=\frac{2 a}{1-<\cos \theta>}
$$

Using random number generators, it is straightforward to generate chains that satisfy this condition $[42,45]$. Since overall conformational features, such as end to end distances, vary greatly from one randomly generated chain to another (when $L$ is significantly larger than $P$ ), it is necessary to average transport results over many different chains in order to obtain good statistics.

At this point, we can ask whether or not it is reasonable to equate average transport properties, such as $\left\langle\Lambda_{l}\right\rangle$, derived from model studies of ensembles of chains "frozen" in their starting configuration, to the actual transport properties of flexible particles. The answer to this question depends, in part, on what is actually measured in a particular experiment. Considerable attention to this point has been given to the "off field" electric birefringence or dichroism decay of duplex DNA [12,37,38,42]. In general, the decay is multi-exponential and consists of end over end tumbling as well as more complex "internal" decay processes [46]. However, provided we are interested in the slowest decay process, which is also the decay process of greatest amplitude for comparatively short DNA fragments, equating $\Lambda_{l}$ in Equation (32) to the average, $\left\langle\Lambda_{l}\right\rangle$, obtained from an ensemble of "frozen" chains, is expected to be an accurate approximation [46].

\subsection{End-Over-End Rotation of Rods}

Figure 2 illustrates the equivalence between the "end-over-end" reduced rotational friction coefficient, $\Lambda_{1}$, of a right circular cylinder and a linear string of touching beads in the absence of a gel $(\lambda=0)$. The solid line represents the right circular cylinder of length $L$ and axial radius $R$ and is computed from Equation (33) [40]. The filled squares, computed using the procedure described in Section 2.1, are for a linear string of $N$ touching beads of radius $a$ with $a / R=1.207$. The length, $L$ is related to $a$ and $N$ by Equation (36) with $c=0.20$.

Figure 2. $\Lambda_{l}$ for a Right Circular Cylinder and Linear String of Touching Beads versus Length. The solid line is for a right circular cylinder of axial radius $R$ and comes from Equation (33) [40]. The filled squares are for a linear string of touching beads of radius $a$ and $a / R=1.207$. The length of the right circular cylinder, $L$, is related to $N$ and $a$ by Equation (36) with $c=0.20$. The gel screening parameter, $\lambda$, is set to zero (no gel).

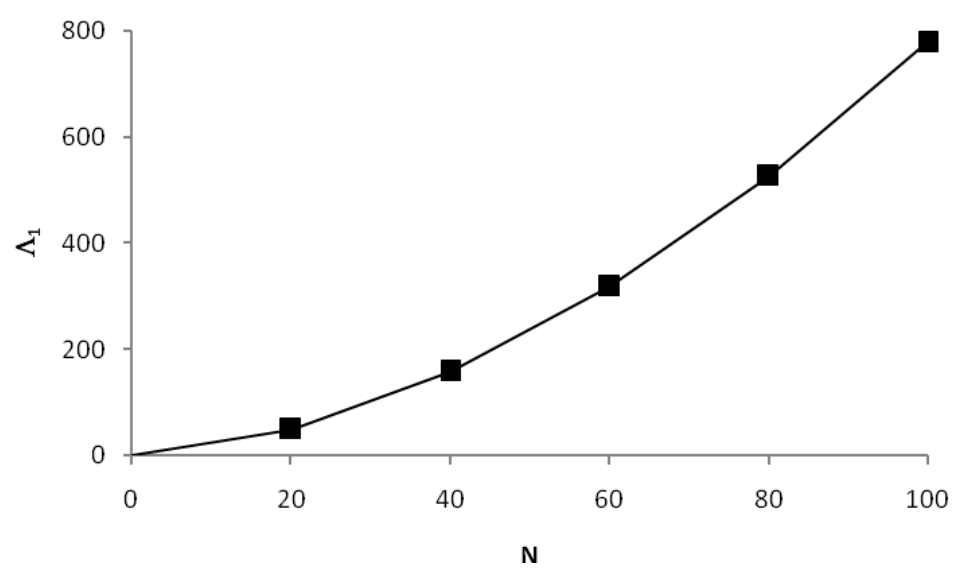


We would next like to consider the effect of the gel on $\Lambda_{l}$. For a sphere of radius $a$ in an EM, it is straightforward to show (using Equations $(6,7,24,26)$ ), that

$$
\frac{\Lambda_{1}}{\Lambda_{1}^{n g}}-1=\frac{\lambda^{2} a^{2}}{3(1+\lambda a)}
$$

where $\Lambda_{l}{ }^{n g}$ is the reduced friction factor for a rotating sphere in the absence of a gel (which equals $8 \pi \eta a^{3}$ ). For a linear string of touching beads (radius $=a$ ), we computed $\Lambda_{l}$ for $N$ ranging from 20 to 100 and $\lambda a$ ranging from 0 to 0.604 using the procedure described in Section 2.1. To within an accuracy of $\pm 2 \%$ over the entire range of $N$ and $\lambda a$, the data can be fit with the following simple semi-empirical form:

$$
\begin{gathered}
\frac{\Lambda_{1}}{\Lambda_{1}^{n g}}-1=d_{1} x^{2}+d_{2} x^{3} \\
x=\ln (1+\gamma) \\
\gamma=\lambda R_{e}=\lambda a\left(N \Lambda_{1}^{n g}\right)^{1 / 3} \\
d_{1}=0.534-0.049 \ln (N)-0.004(\ln (N))^{2} \\
d_{2}=-0.001
\end{gathered}
$$

Above, $\Lambda_{l}{ }^{n g}$ represents the reduced eigenvalue of the bead array in the absence of a gel $(\lambda=0)$, and $R_{e}$ is the radius of a sphere that has the same reduced rotational friction coefficient as the bead array in the absence of a gel.

\section{Experimental Section: Rotational Relaxation of DNA in a Dilute Agarose Gel}

Stellwagen [12] has reported longest lifetime, $\tau_{l}$, "off field" electric birefringence decays of 622 base pair DNA in dilute agarose gels with $\mathrm{M}$ varying from 0 to $0.015 \mathrm{gm}$ "dry" gel $/ \mathrm{mL}$. The experiments were carried out at $20^{\circ} \mathrm{C}$ in $0.2 \mathrm{mM}$ aqueous Tris buffer at a $\mathrm{pH}$ of around 8.0. A discrete wormlike chain model consisting of 88 touching beads was used to model 622 bp DNA following the procedure discussed in Section 2.2. Figure 3 shows a representative discrete wormlike chain comprised of 88 beads with $P=65 \mathrm{~nm}$. For structures such as these, reduced rotational eigenvalues, $\Lambda_{l}{ }^{\exp }$, corresponding to reported lifetimes, $\tau_{l}$, can be determined using Equation (32). The relative uncertainty in these experimental numbers is estimated to be approximately $3 \%$. For each simulation, 300 wormlike chain configurations were generated at random and an average $\Lambda_{l}$ was computed. We chose 300 configurations in order to obtain relative model uncertainties in $\left\langle\Lambda_{l}\right\rangle$ that are accurate to about $3 \%$. 
Figure 3. A 88 Subunit Discrete Wormlike Chain. The persistence length, $P$, is $65 \mathrm{~nm}$ and the bead radius, $a$, is $1.207 \mathrm{~nm}$. Different configurations are generated at random as discussed in the text.

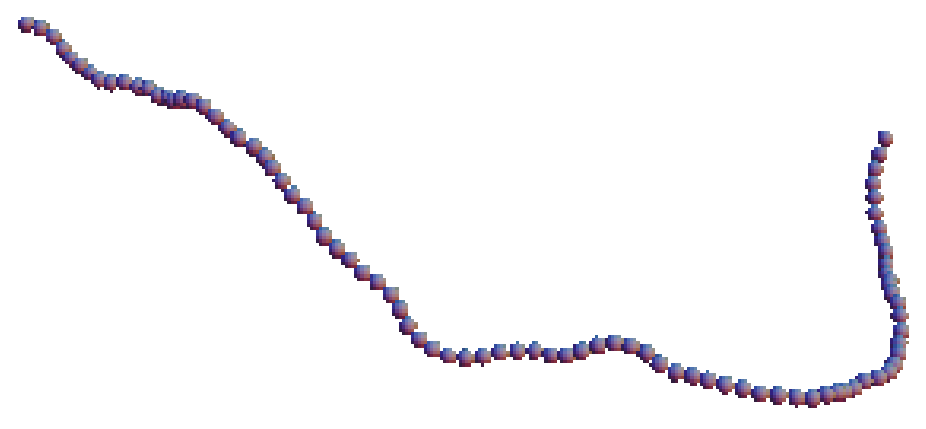

There are two model parameters that need to be determined and they are the persistence length of the DNA, $P$, and the average gel fiber radius, $r_{f}$ (see Equation (3)). $P$ can be determined by varying this quantity in modeling until $\Lambda_{l}^{\text {exp }}$ and $\left\langle\Lambda_{l}\right\rangle$ match (317). In the absence of a gel, $\Lambda_{l}^{\text {exp }}$ is best fit by setting $P=65 \mathrm{~nm}$ in modeling. This value is in good agreement with experiment when the comparatively low salt conditions of the experiment are factored in [44]. Under physiological salt conditions, a value of $P$ of around $50 \mathrm{~nm}$ is expected. Figure 4 summarizes our comparison of $\Lambda_{l}^{\exp }$ and model $\Lambda_{l}$. Filled squares denote experimental values and the solid, dotted, and dashed lines represent $r_{g}=1.52,2.0$, and $2.5 \mathrm{~nm}$, respectively. X-ray diffraction studies of agarose indicate a significant population of fibers with $r_{g}$ of about $1.52 \mathrm{~nm}$, but a fraction of significantly thicker fibers [47]. This motivated the choice of $1.52 \mathrm{~nm}$. The choice of the higher values comes from fitting the electrophoretic mobility of Au nanoparticles in agarose gels [15]. From Figure 4, a gel fiber radius of about $2.5 \mathrm{~nm}$ appears to be most consistent with experiment. Although this $\mathrm{r}_{\mathrm{g}}$ value along with $P=65 \mathrm{~nm}$ for DNA appears to describe well the rotational relaxation behavior of DNA in dilute gels $(M \leq 0.10$ gm "dry" gel $/ \mathrm{mL})$, it substantially underestimates the rotational lifetimes in more concentrated gels.

Figure 4. Model and Experimental $\Lambda_{l}$ Values for 622 bp DNA as a Function of Gel Concentration. Experimental values are denoted by the filled squares. Solid, dotted, and dashed lines denote model studies with $P=65 \mathrm{~nm}$ and $r_{g}=1.52,2.0$, and $2.5 \mathrm{~nm}$, respectively. The temperature is $20^{\circ} \mathrm{C}$.

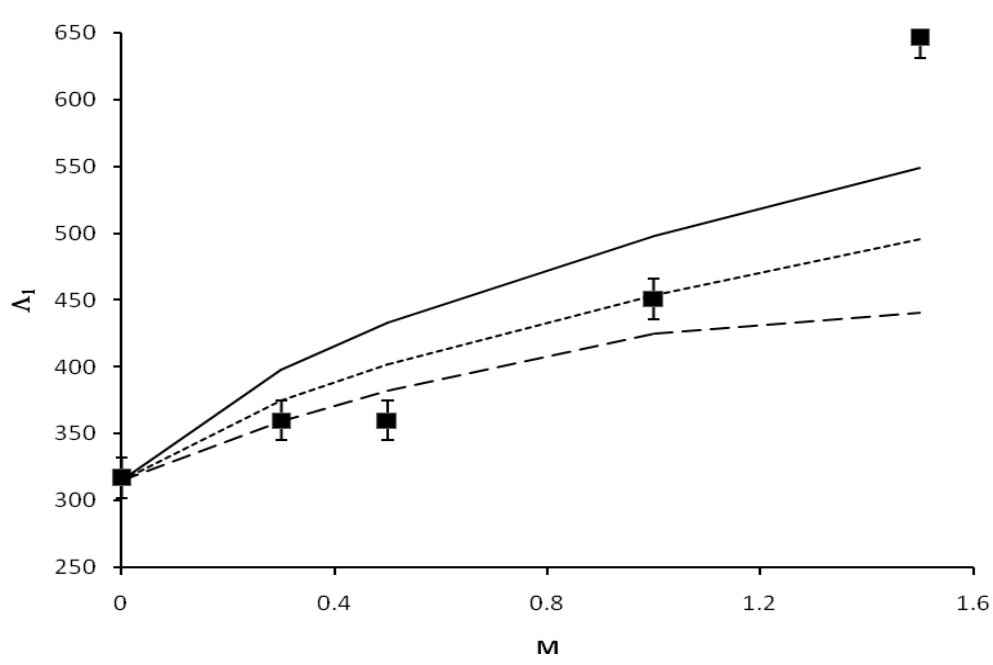


The EM model that we have used accounts for long range hydrodynamic interactions, HI, but ignores direct interactions. If a characteristic length of our macromolecule is much smaller than the average spacing between gel fibers, $A$, then direct interactions would not be expected to significantly retard the random rotational motion of our macromolecule. For flexible duplex DNA modeled as a wormlike chain, we can set this "characteristic length" equal to the persistence length, $P$. If a dilute gel is modeled as a simple cubic lattice of gel fibers, then [48]

$$
A \cong r_{g} \sqrt{\frac{3 \pi \rho_{g} \omega_{s}}{M}}
$$

See the discussion following Equation (3) for the definitions of $\rho_{g}$ and $\omega_{s}$ and their values for agarose. If we set $r_{g}$ equal to $2.5 \mathrm{~nm}$, then $A=142,110,78$, and $63 \mathrm{~nm}$ for $M=0.003,0.005,0.010$, and $0.015 \mathrm{gm} / \mathrm{mL}$, respectively. It is important to note that A decreases with increasing $\mathrm{M}$ and becomes comparable to $\mathrm{P}$ for $\mathrm{M}$ in the 0.010 to 0.015 range. For $M \leq 0.010 \mathrm{gm} / \mathrm{mL}$, the gel fiber spacing is comparatively large and long range hydrodynamic interaction is the dominant interaction. For $M>0.010 \mathrm{gm} / \mathrm{mL}$, however, direct interactions become important.

\section{Conclusions}

The objective of the present study is to apply the Effective Medium (EM) model to the rotational motion of a macromolecule modeled as an array of non-overlapping beads and then apply it to several cases including duplex DNA in agarose gels. This is an extension of earlier work which focused on the translational motion of similar model macromolecules in an EM [16]. The presence of a gel retards the rotational motion of a particle in a gel and this retardation is due to both long range hydrodynamic interaction, HI, and (short range) direct interactions. EM modeling accounts for long range HI, but not direct interactions. The EM modeling procedure is used to examine the electric birenfringence decay of a 622 base pair DNA fragments in an agarose gel [12]. At low gel concentration $(M \leq 0.010 \mathrm{gm} / \mathrm{mL})$ where long range interactions dominate, good agreement between theory and experiment is achieved if the persistence length, $P$, of DNA is taken to be $65 \mathrm{~nm}$ and the gel fiber radius, $r_{g}$, of agarose is taken to be $2.5 \mathrm{~nm}$. A persistence length of this magnitude is consistent with independent studies [44]. Also, a gel fiber radius of $2.5 \mathrm{~nm}$ is consistent with earlier modeling studies of DNA diffusion [16] as well as the electrophoresis of $\mathrm{Au}$ nanoparticles [15] in agarose gels. At higher gel concentrations, the EM model substantially underestimates the rotational relaxation time of DNA. By means of simple modeling, we have shown that short range interactions become important when the average gel fiber spacing, $A$, becomes comparable to $P$.

It has been recognized for some time that the behavior of macromolecules in gel electrophoresis fall into well defined "regimes" [49], and the findings of the present study are consistent with this view. At low gel concentration where long range HI dominates, the EM model is adequate, but this breaks down at high gel concentration where reptation theories are undoubtedly more appropriate [50]. In the case of rotational motion, it appears as though the transition between "long range" and "short range" regimes occurs over a narrow interval of gel concentration and that the nature of this rotational motion could be very different in the two regimes. More experimental and modeling studies are required to address this issue. It would also be possible to extend these studies to circular duplex DNA in gels as a 
function of linking number [51]. Conformations could be generated using established Brownian dynamics procedures $[28,45,51]$ followed by application of the methodology of the present work to account for the long range hydrodynamic effect of the gel.

\section{References}

1. Zimmerman, S.B.; Minton, A. Macromolecular crowding: Biochemical, biophysical, and physiological consequences. Annu. Rev. Biophys. Biomol. Struct. 1993, 22, $27-65$.

2. Dwyer, J.D.; Bloomfield, V.A. Brownian dynamics simulations of probe and self-diffusion in concentrated protein and DNA solutions. Biophys. J. 1993, 65, 1810-1816.

3. Johnson, E.M.; Berk, D.A.; Jain, R.K.; Deen, W.M. Hindered diffusion in agarose gels: Test of effective medium model. Biophys. J. 1996, 70, 1017-1026.

4. Pluen, A.; Netti, P.A.; Jain, R.K.; Deen, W.M. Diffusion of macromolecules in agarose gels: Comparison of linear and globular configurations. Biophys. J. 1999, 77, 542-552.

5. Pernodet, N.; Tinland, B.; Sturm, J.; Weil, G. Brownian diffusion and electrophoretic transport of double-stranded DNA in agarose gels. Biopolymers 1999, 50, 45-59.

6. Sass, H.J.; Musco, G.; Stahl, S.J.; Wingfield, P.T.; Grzesiek, S. Solution NMR of proteins within polyacrylamide gels: Diffusional properties and residual alignment by mechanical stress or embedding of oriented purple membranes. J. Biomol. NMR 2000, 18, 303-309.

7. Fatin-Rouge, N.; Starchev, K.; Buffle, J. Size effects on diffusion processes within agarose gels. Biophys. J. 2004, 86, 2710-2719.

8. Tatarkova, S.A.; Berk, D.A. Probing single DNA mobility with fluorescence correlation spectroscopy. Phys. Rev. E 2005, 71, 41913-1-41913-5.

9. Hirota, N.; Kumaki, Y.; Narita, T.; Gong, J.P.; Osada, Y. Effect of charge on protein diffusion in hydrogels. J. Phys. Chem. B 2000, 104, 9898-9903.

10. Fatin-Rouge, N.; Milon, A.; Buffle, J. Diffusion and partitioning of solutes in agarose hydrogels: The relative influence of electrostatic and specific interactions. J. Phys. Chem. B 2003, 107, 12126-12137.

11. Wijmenga, S.S.; Maxwell, A. Rotational diffusion of short DNA fragments in polyacrylamide gels: An electric birefringence study. Biopolymers 1986, 25, 2173-2186.

12. Stellwagen, N.C. The use of transient electric birefringence to characterize the conformation of DNA in solution, the mechanism of DNA gel electrophoresis, and the structure of agarose gels. Colloids Surf. A 2002, 209, 107-122.

13. Hungerford, G.; Rei, A.; Ferreira, M.I.C.; Suhling, K.; Tregido, K. Diffusion in a sol-gel-derived medium with a view toward biosensor applications. J. Phys. Chem. B 2007, 111, 3558-3562.

14. Shen, H.; Hu, Y.; Saltzman, W.M. DNA diffusion in mucus: Effect of size, topology of DNAs, and transfection reagents. Biophys. J. 2006, 91, 639-644.

15. Allison, S.A.; Xin, Y.; Pei, H. Electrophoresis of spheres with uniform zeta potential in a gel modeled as an effective medium. J. Coll. Interface Sci. 2007, 313, 328-337.

16. Allison, S.A.; Pei, H.; Haynes, B.M.H.; Xin, Y.; Law, L.; Labrun, J.; Augustin, D. Translational diffusion of macromolecules and nanoparticles modeled as non overlapping bead arrays in an effective medium. J. Phys. Chem. B 2008, 112, 5858-5866. 
17. Pei, H.; Allison, S.A.; Haynes, B.M.H.; Augustin, D. Brownian dynamics simulation of the diffusion of rods and wormlike chains in a gel modeled as a cubic lattice: Application to DNA. $J$. Phys. Chem. B 2009, 113, 2564-2571.

18. Brinkman, H.C. A calculation of the viscous force exerted by a flowing fluid on a dense swarm of particles. Appl. Sci. Res. A 1947, 1, 27-34.

19. Debye, P.; Bueche, A.M. Intrinsic viscosity, diffusion, and sedimentation rate of polymers in solution. J. Chem. Phys. 1948, 16, 573-579.

20. Felderhof, B.U.; Deutch, J.M. Frictional properties of dilute polymer solutions. I. Rotational friction coefficient. J. Chem. Phys. 1975, 62, 2391-2397.

21. Felderhof, B.U. Frictional properties of dilute polymer solutions. III. Translational friction coefficient. Physica 1975, 80A, 63-75.

22. Allison, S.A.; Pei, Y.; Xin, Y. Review: Modeling the free solution and gel electrophoresis of biopolymers: The bead array-effective medium model. Biopolymers 2007, 87, 102-114.

23. Stigter, D. Influence of agarose gel on electrophoretic stretch, on trapping, and on relaxation of DNA. Macromolecules 2000, 33, 8878-8889.

24. Ferree, S.; Blanch, H.W. The hydrodynamics of DNA electrophoretic stretch and relaxation in a polymer solution. Biophys. J. 2004, 87, 468-475.

25. Jackson, G.W.; James, D.G. The permeability of fibrous porous media. Can. J. Chem. 1986, 64, 362-374.

26. Laurent, T.C. Determination of the structure of agarose gels by gel chromatography. Biochim. Biophys. Acta 1967, 136, 199-205.

27. Johnson, E.M.; Berk, D.A.; Jain, R.K. Diffusion and partitioning of proteins in charged agarose gels. Biophys. J. 1995, 68, 1561-1568.

28. Allison, S.A. Boundary element modeling of biomolecular transport. Biophys. Chem. 2001, 93, 197-213.

29. Happel, J.; Brenner, H. Low Reynolds Number Hydrodynamics; Martinus Nijhoff: The Hague, The Netherlands, 1983.

30. Kim, S.; Karilla, S.J. Microhydrodynamics; Butterworth-Heinemann: Boston, MA, USA, 1991.

31. Garcia de la Torre, J.; Rodes, V. Effects from bead size and hydrodynamic interactions on the translational and rotational coefficients of macromolecular bead models. J. Chem. Phys. 1983, 79, 2454-2460.

32. Garcia de la Torre, J.; Bloomfield, V.A. Hydrodynamic properties of complex, rigid, biological macromolecules: Theory and applications. Quart. Rev. Biophys. 1981, 14, 81-139.

33. Garcia de la Torre, J.; Huertas, M.L.; Carrasco, B. Calculation of hydrodynamic properties of globular proteins from their atomic level structure. Biophys. J. 2000, 78, 719-730.

34. Aragon, S.R. A precise boundary element method for macromolecular transport properties. J. Comput. Chem. 2004, 25, 1191-1205.

35. Garcia Bernal, J.M.; Garcia de la Torre, J. Transport properties and hydrodynamic centers of rigid macromolecules with arbitrary shape. Biopolymers 1980, 19, 751-766.

36. Garcia de la Torre, J.; Jimeniz, A.; Freire, J.J. Monte Carlo calculation of hydrodynamic properties of freely rotating, and real polymethylene chains. Macromolecules 1982, 15, 148-154. 
37. Elias, J.G.; Eden, D. Transient electric birefringence study of the persistence length and electrical polarizability of restriction fragments of DNA. Macromolecules 1981, 14, 410-419.

38. Hagerman, P.J. Investigation of the flexibility of DNA using transient electric birefringence. Biopolymers 1981, 20, 1503-1535.

39. Diekmann, S.; Hillen, W.; Morgeneyer, B.; Wells, R.D.; Porschke, D. Orientation relaxation of DNA restriction fragments and the internal mobility of the double helix. Biophys. Chem. 1982, 15, 263-270.

40. Tirado, M.M.; Martinez, C.M.; Garcia de la Torre, J. Comparison of theories for the translational and rotational diffusion coefficients of rodlike macromolecules. Application to short DNA restriction fragments. J. Chem. Phys. 1984, 81, 2047-2052.

41. Eimer, W.; Williamson, J.R.; Boxer, S.G.; Pecora, R. Characterization of the overall and internal dynamics of short oligonucleotides by depolarized dynamic light scattering and NMR relaxation measurements. Biochemistry 1990, 29, 799-811.

42. Hagerman, P.J.; Zimm, B.H. Monte Carlo approach to the analysis of the rotational diffusion of wormlike chains. Biopolymers 1981, 20, 1481-1502.

43. Schellman, J.A.; Harvey, S.C. Static contributions to the persistence length of DNA and dynamic contributions to DNA curvature. Biophys. Chem. 1995, 55, 95-114.

44. Geggier, S.; Kotlyar, A.; Vologodskii, A. Temperature dependence of DNA persistence length. Nucl. Acids Res. 2010, 39, 1419-1426.

45. Allison, S.A.; Schurr, J.M. Effect of regular anisotropic permanent bending on the diffusional spinning and fluorescence polarization anisotropy of short DNA fragments studied by Brownian dynamics simulation. Macromolecules 1997, 30, 7131-7142.

46. Lewis, R.J.; Allison, S.A.; Eden, D.; Pecora, R. Brownian dynamics simulations of a three-subunit and a ten-subunit wormlike chain: Comparison of results with trumbell theory and with experimental results from DNA. J. Chem. Phys. 1988, 89, 2490-2503.

47. Djabourov, M.; Clark, A.H.; Rowlands, D.W.; Murphy, S.B. Small angle X-ray scattering characterization of agarose gels and sols. Macromolecules 1989, 22, 180-188.

48. Allison, S.A.; Li, Z.; Reed, D.; Stellwagen, N.C. Modeling the gel electrophoresis of short duplex DNA by Brownian dynamics: Cubic cell lattice with direct interaction. Electrophoresis 2002, 23, 2678-2689.

49. Calladine, C.R.; Collis, C.M.; Drew, H.R.; Mott, M.R. A study of electrophoretic mobility of DNA in agarose and polyacrylamide gels. J. Mol. Biol. 1991, 221, 981-1005.

50. Zimm, B.H.; Levene, S.D. Problems and prospects in the theory of gel electrophoresis of DNA. Quart. Rev. Biophys. 1992, 25, 171-204.

51. Chirico, G.; Langowski, J. Brownian dynamics simulation of supercoiled DNA with bent sequences. Biophys. J. 1996, 71, 955-971.

(C) 2011 by the authors; licensee MDPI, Basel, Switzerland. This article is an open access article distributed under the terms and conditions of the Creative Commons Attribution license (http://creativecommons.org/licenses/by/3.0/). 